\title{
ON THE WEAK AND ERGODIC LIMIT OF THE SPECTRAL SHIFT FUNCTION
}

\author{
V. BOROVYK AND K. A. MAKAROV
}

\begin{abstract}
We discuss convergence properties of the spectral shift functions associated with a pair of Schrödinger operators with Dirichlet boundary conditions at the end points of a finite interval $(0, r)$ as the length of interval approaches infinity.
\end{abstract}

\section{INTRODUCTION}

In this note we study the relationship between the spectral shift function $\xi$ associated with the pair $\left(H_{0}, H\right)$ of the half-line Dirichlet Schrödinger operators

$$
H_{0}=-\frac{d^{2}}{d x^{2}} \quad \text { and } \quad H=-\frac{d^{2}}{d x^{2}}+V(x) \quad \text { in } L^{2}(0, \infty)
$$

and the spectral shift function $\xi^{r}$ associated with the pair $\left(H_{0}^{r}, H^{r}\right)$ of the corresponding Schrödinger operators on the finite interval $(0, r)$ with the Dirichlet boundary conditions at the end points of the interval.

Recall that given a pair of self-adjoint operators $\left(H_{0}, H\right)$ in a separable Hilbert space $\mathcal{H}$ such that the difference $(H+i I)^{-1}-\left(H_{0}+i I\right)^{-1}$ is a trace class operator, the spectral shift function $\xi$ associated with the pair $\left(H_{0}, H\right)$ is uniquely determined (up to an additive constant) by the trace formula

$$
\operatorname{tr}\left(f(H)-f\left(H_{0}\right)\right)=\int_{\mathbb{R}} \xi(\lambda) f^{\prime}(\lambda) d \lambda,
$$

valid for a wide class of functions $f([3],[4],[10],[11])[15])$.

In the case where both $H_{0}$ and $H$ are bounded from below, the standard way to fix the undetermined constant is to require that

$$
\xi(\lambda)=0 \quad \text { for } \quad \lambda<\inf \left\{\operatorname{spec}\left(H_{0}\right) \cup \operatorname{spec}(H)\right\} .
$$

Under the short range hypothesis on the potential $V$ that

$$
\int_{0}^{\infty}(1+x)|V(x)| d x<\infty
$$

the pairs of the half-line Schrödinger operators $\left(H_{0}, H\right)$ and their finite-interval box-approximations $\left(H_{0}^{r}, H^{r}\right)$ are resolvent comparable and the spectral shift functions $\xi$ and $\xi^{r}$ associated with the pairs $\left(H_{0}, H\right)$ and $\left(H_{0}^{r}, H^{r}\right)$ satisfying the normalization condition (1.2) are well-defined. The specifics of the one-dimensional case is that each of the spectral shift functions $\xi$ and $\xi^{r}$ admits a unique left-continuous representative, for which we will keep the same notation.

The main result of this note is the following theorem.

1991 Mathematics Subject Classification. Primary: 81Q10, Secondary: 35P20, 47N50.

Key words and phrases. Spectral shift function, scattering phase, Jost function, Schrödinger operators. 
Theorem 1. Assume that a real-valued function $V$ on $(0, \infty)$ satisfies condition (1.3). Denote by $\xi$ and $\xi^{r}$ the left-continuous spectral shift functions associated with the pairs $\left(H_{0}, H\right)$ and $\left(H_{0}^{r}, H^{r}\right)$ of the Dirichlet Schrödinger operators on the semi-axis $(0, \infty)$ and on the finite interval $(0, r)$, respectively.

Then for any continuous function $g$ on $\mathbb{R}$ with compact support both the (weak) limit

$$
\lim _{r \rightarrow \infty} \int_{-\infty}^{\infty} \xi^{r}(\lambda) g(\lambda) d \lambda=\int_{-\infty}^{\infty} \xi(\lambda) g(\lambda) d \lambda
$$

and the Cesàro limit

$$
\lim _{r \rightarrow \infty} \frac{1}{r} \int_{0}^{r} \xi^{r}(\lambda) d r=\xi(\lambda), \quad \lambda \in \mathbb{R} \backslash\left(\operatorname{spec}_{d}(H) \cup\{0\}\right)
$$

exist.

If, in addition, the Schrödinger operator $H=-\frac{d^{2}}{d x^{2}}+V(x)$ has no zero energy resonance, then convergence (1.5) takes place for $\lambda=0$ as well.

We remark that the weak convergence result (1.4) has been obtained in [9] in the case of arbitrary dimension under the assumption that the potential $V$ belongs to the BirmanSolomyak class $\ell^{1}\left(L^{2}\right)$. In contrast to the Feymann-Kac path integration approach developed in [9], our approach is based on the study of fine properties of the eigenvalue counting function available in the one-dimensional case.

We also remark that the class of potentials satisfying condition 1.3 is slightly different from the Birman-Solomyak class $\ell^{1}\left(L^{2}\right)$ : the Birman-Solomyak condition allows a slower decay at infinity, while condition (1.3) admits a $L^{1}$-type singularities at finite points in contrast to the fact that only $L^{2}$-type singularities are allowed in the Birman-Solomyak class.

\section{SOME GENERAL CONVERGENCE RESULtS}

In this section we will develop the necessary analytic background for proving the convergence results (1.4) and (1.5).

Assume the following hypothesis.

Hypothesis 2.1. Assume that $f$ is a Riemann integrable function on the interval $[0,1]$ and $g$ is a continuous function on $[0,1]$. Suppose that a sequence of real-valued measurable functions $\left\{f_{n}\right\}_{n=1}^{\infty}$ on $[0,1]$ converges to $f$ pointwise on the open interval $(0,1)$ and that the convergence is uniform on every compact set of the semi-open interval $(0,1]$. Assume, in addition, that the sequence $\left\{f_{n}\right\}_{n=1}^{\infty}$ has a $|g|$-integrable majorante $F$. That is,

$$
\left|f_{n}(x)\right| \leq F(x), \quad x \in[0,1], n \in \mathbb{N},
$$

and

$$
\int_{0}^{1} F(x)|g(x)| d x<\infty .
$$

Our main technical result is the following lemma.

Lemma 2.2. Assume Hypothesis 2.1 Suppose that $\left\{r_{n}\right\}_{n=1}^{\infty}$ is a sequence of non-negative numbers such that

$$
\lim _{n \rightarrow \infty} r_{n}=\infty
$$


Then

$$
\lim _{n \rightarrow \infty} \int_{0}^{1} \tilde{f}_{n}(x) g(x) d x=\int_{0}^{1} f(x) g(x) d x,
$$

where the sequence $\left\{\tilde{f}_{n}\right\}_{n=1}^{\infty}$ is given by

$$
\tilde{f}_{n}(x)=\left[r_{n} x+f_{n}(x)\right]-\left[r_{n} x\right], \quad n \in \mathbb{N}, \quad x \in[0,1],
$$

and $[\cdot]$ stands for the integer value function.

Proof. Without loss of generality one may assume that the function $g$ in non-negative on $[0,1]$.

A simple change of variables shows that

$$
\begin{aligned}
\int_{0}^{1} \tilde{f}_{n}(x) g(x) d x=\frac{1}{r_{n}} \int_{0}^{r_{n}}\left(\left[t+f_{n}\left(r_{n}^{-1} t\right)\right]-[t]\right) g\left(r_{n}^{-1} t\right) d x \\
\quad=\sum_{k=0}^{\left[r_{n}\right]-1} \frac{1}{r_{n}} \int_{k}^{k+1}\left(\left[t+f_{n}\left(r_{n}^{-1} t\right)\right]-[t]\right) g\left(r_{n}{ }^{-1} t\right) d t+\varepsilon_{n} \\
=\sum_{k=0}^{\left[r_{n}\right]-1} \frac{1}{r_{n}} \int_{0}^{1}\left(\left[t+k+f_{n}\left(\frac{t+k}{r_{n}}\right)\right]-[t+k]\right) g\left(\frac{t+k}{r_{n}}\right) d t+\varepsilon_{n}
\end{aligned}
$$

where

$$
\varepsilon_{n}=\int_{\frac{\left[r_{n}\right]}{r_{n}}}^{1} \widetilde{f}_{n}(x) g(x) d x .
$$

Taking into account that $[t+k]=[t]$ for any $t$ whenever $k$ is an integer, and $[t]=0$ for $t \in[0,1)$, we get

$$
\int_{0}^{1} \tilde{f}_{n}(x) g(x) d x=\sum_{k=0}^{\left[r_{n}\right]-1} \frac{1}{r_{n}} \int_{0}^{1}\left[t+f_{n}\left(\frac{t+k}{r_{n}}\right)\right] g\left(\frac{t+k}{r_{n}}\right) d t+\varepsilon_{n} .
$$

The uniform bound $\left|\widetilde{f}_{n}(x)\right| \leq F(x)+1$ (with $F$ from 2.1) shows that

$$
\left|\varepsilon_{n}\right| \leq \int_{\frac{\left[r_{n}\right]}{r_{n}}}^{1}(F(x)+1) g(x) d x, \quad n \in \mathbb{N}
$$

and therefore

$$
\lim _{n \rightarrow \infty} \varepsilon_{n}=0
$$

Combining (2.6) and (2.7) with the estimate

$$
\left|\int_{0}^{1}\left[t+f_{n}\left(\frac{t+k}{r_{n}}\right)\right]\left(g\left(\frac{t+k}{r_{n}}\right)-g\left(\frac{k}{r_{n}}\right)\right) d t\right| \leq\left(\left\|f_{n}\right\|_{\infty}+1\right) \omega_{g}\left(r_{n}^{-1}\right),
$$

where $\omega_{g}(\cdot)$ stands for the modulus of continuity of the function $g$, one concludes that

$$
\lim _{n \rightarrow \infty} \int_{0}^{1} \tilde{f}_{n}(x) g(x) d x=\lim _{n \rightarrow \infty} \sum_{k=0}^{\left[r_{n}\right]-1} \frac{g\left(k r_{n}^{-1}\right)}{r_{n}} \int_{0}^{1}\left[t+f_{n}\left(\frac{t+k}{r_{n}}\right)\right] d t
$$


(provided that the limit in the RHS exists.) Therefore, in order to prove assertion (2.3) it suffices to establish the equality

$$
\lim _{n \rightarrow \infty} \sum_{k=0}^{\left[r_{n}\right]-1} \frac{g\left(k r_{n}^{-1}\right)}{r_{n}} \int_{0}^{1}\left[t+f_{n}\left(\frac{t+k}{r_{n}}\right)\right] d t=\int_{0}^{1} f(x) g(x) d x .
$$

To prove (2.9), assume temporarily that the sequence of functions $\left\{f_{n}\right\}_{n=1}^{\infty}$ converges to $f$ uniformly on the closed interval $[0,1]$.

Since the function $t \mapsto[t]$ is monotone, from the inequality

$$
\begin{gathered}
t+\inf _{\left[\frac{k}{r_{n}}, \frac{k+1}{r_{n}}\right]} f-\delta_{n} \leq t+f_{n}\left(\frac{t+k}{r_{n}}\right) \leq t+\sup _{\left[\frac{k}{r_{n}}, \frac{k+1}{r_{n}}\right]} f+\delta_{n}, \\
t \in[0,1], \quad k=0,1, \ldots,\left[r_{n}\right]-1,
\end{gathered}
$$

where $\delta_{n}=\left\|f-f_{n}\right\|_{\infty}$, one immediately obtains that

$$
\begin{gathered}
{\left[t+\inf _{\left[\frac{k}{r_{n}}, \frac{k+1}{r_{n}}\right]} f-\delta_{n}\right] \leq\left[t+f_{n}\left(\frac{t+k}{r_{n}}\right)\right] \leq\left[t+\sup _{\left[\frac{k}{r_{n}}, \frac{k+1}{r_{n}}\right]} f+\delta_{n}\right],} \\
t \in[0,1], \quad k=0,1, \ldots,\left[r_{n}\right]-1 .
\end{gathered}
$$

Integrating (2.10) against $t$ from 0 to 1 and noticing that

$$
\int_{0}^{1}[t+a] d t=a, \quad \text { for all } a \in \mathbb{R}
$$

and that $g$ is a non-negative function, one obtains the following two-sided estimate

$$
\begin{aligned}
\sum_{k=0}^{\left[r_{n}\right]-1} \frac{g\left(\frac{k}{r_{n}}\right)}{r_{n}}\left(\inf _{\left[\frac{k}{r_{n}}, \frac{k+1}{r_{n}}\right]} f-\delta_{n}\right) \leq & \sum_{k=0}^{\left[r_{n}\right]-1} \frac{g\left(\frac{k}{r_{n}}\right)}{r_{n}} \int_{0}^{1}\left[t+f_{n}\left(\frac{t+k}{r_{n}}\right)\right] d t \\
& \leq \sum_{k=0}^{\left[r_{n}\right]-1} \frac{g\left(\frac{k}{r_{n}}\right)}{r_{n}}\left(\sup _{\left[\frac{k}{r_{n}}, \frac{k+1}{r_{n}}\right]} f+\delta_{n}\right) .
\end{aligned}
$$

Since $f$ is a Riemann integrable function by hypothesis and the function $g$ is continuous on $[0,1]$, one concludes that

$$
\begin{gathered}
\lim _{n \rightarrow \infty} \frac{1}{r_{n}} \sum_{k=0}^{\left[r_{n}\right]-1} g\left(k r_{n}^{-1}\right) \inf _{\left[\frac{k}{r_{n}}, \frac{k+1}{r_{n}}\right]} f=\lim _{n \rightarrow \infty} \frac{1}{r_{n}} \sum_{k=0}^{\left[r_{n}\right]-1} g\left(k r_{n}^{-1}\right) \sup _{\left[\frac{k}{r_{n}}, \frac{k+1}{r_{n}}\right]} f \\
=\int_{0}^{1} f(x) g(x) d x .
\end{gathered}
$$

The additional assumption that the sequence $\left\{f_{n}\right\}_{n=1}^{\infty}$ converges to $f$ uniformly on the closed interval $[0,1]$ means that

$$
\lim _{n \rightarrow \infty} \delta_{n}=0,
$$

which together with (2.11) and (2.12) proves (2.9). This completes the proof of (2.3) (provided that $\left\{f_{n}\right\}_{n=1}^{\infty}$ converges to $f$ uniformly on the $[0,1]$ ).

To remove the extra assumption, we proceed as follows.

Given $0<\varepsilon<1$, one gets the inequality

$$
\varlimsup_{n \rightarrow \infty}\left|\int_{0}^{1} \widetilde{f}_{n}(x) g(x) d x-\int_{0}^{1} f(x) g(x) d x\right|
$$




$$
\begin{gathered}
\leq \varlimsup_{n \rightarrow \infty}\left|\int_{\varepsilon}^{1} \tilde{f}_{n}(x) g(x) d x-\int_{\varepsilon}^{1} f(x) g(x) d x\right| \\
+\int_{0}^{\varepsilon}(2 F(x)+1) g(x) d x .
\end{gathered}
$$

By hypothesis the sequence $\left\{f_{n}\right\}_{n=1}^{\infty}$ converges uniformly on the interval $(\varepsilon, 1]$ and therefore by the first part of the proof one concludes that

$$
\lim _{n \rightarrow \infty}\left|\int_{\varepsilon}^{1} \tilde{f}_{n}(x) g(x) d x-\int_{\varepsilon}^{1} f(x) g(x) d x\right|=0 .
$$

Combining (2.13) and 2.14) one obtains the inequality

$$
\varlimsup_{n \rightarrow \infty}\left|\int_{0}^{1} \tilde{f}_{n}(x) g(x) d x-\int_{0}^{1} f(x) g(x) d x\right| \leq \int_{0}^{\varepsilon}(2 F(x)+1)|g(x)| d x .
$$

By the second inequality in (2.3), the right hand side of (2.15) can be made arbitrary small by an appropriate choice of $\varepsilon$ and hence

$$
\lim _{n \rightarrow \infty}\left|\int_{0}^{1} \tilde{f}_{n}(x) g(x) d x-\int_{0}^{1} f(x) g(x) d x\right|=0
$$

which completes the proof of the lemma.

Corollary 2.3. Let h be a real-valued measurable bounded measurable function on $[0, \infty)$. Suppose that the limit

$$
A=\lim _{x \rightarrow \infty} h(x)
$$

exists. Then

$$
\lim _{r \rightarrow \infty} \frac{1}{r} \int_{0}^{r}([x+h(x)]-[x]) d x=A .
$$

Proof. We will prove that convergence 2.16 holds as $r$ approaches infinity along an arbitrary sequence $\left\{r_{n}\right\}_{n=1}^{\infty}$ of positive numbers such that $\lim _{n \rightarrow \infty} r_{n}=\infty$.

Introduce the sequence of functions $\left\{f_{n}\right\}_{n=1}^{\infty}$ by

$$
f_{n}(x)=h\left(r_{n} x\right), \quad x \in[0,1] .
$$

The sequence $\left\{f_{n}\right\}_{n=1}^{\infty}$ converges pointwise to a constant function $f$ given by

$$
A=\lim _{x \rightarrow \infty} h(x)
$$

and, moreover, the convergence is uniform on every compact set of the semi-open interval $(0,1]$. Since, by hypothesis, the function $h$ is bounded, so is the sequence $\left\{f_{n}\right\}_{n=1}^{\infty}$ and hence one can apply Lemma 2.2 to conclude that

$$
\lim _{n \rightarrow \infty} \int_{0}^{1}\left(\left[r_{n} x+f_{n}(x)\right]-\left[r_{n} x\right]\right) d x=\int_{0}^{1} A d x=A .
$$

By a change of variables one gets

$$
\frac{1}{r_{n}} \int_{0}^{r_{n}}([x+h(x)]-[x]) d x=\int_{0}^{1}\left(\left[r_{n} x+f_{n}(x)\right]-\left[r_{n} x\right]\right) d x,
$$

which together with 2.17 proves that

$$
\lim _{n \rightarrow \infty} \frac{1}{r_{n}} \int_{0}^{r_{n}}([x+h(x)]-[x]) d x=A
$$

and hence (2.16) holds, since $\left\{r_{n}\right\}_{n=1}^{\infty}$ is an arbitrary sequence. 


\section{Proof of TheOREM 1}

Proof. As it easily follows from the trace formula (1.1), the value of the spectral shift function $\xi(\lambda)$ on the negative semi-axis associated with the pair $\left(H_{0}, H\right)$ of the (Dirichlet) Schrödinger operators is directly linked to the number of negative eigenvalues of the operator $H$ that are smaller than $\lambda$,

$$
\xi(\lambda)=-N(\lambda), \quad \lambda<0 .
$$

Analogously, the spectral shift function $\xi^{r}$ associated with the finite-interval Schrödinger operators $\left(H_{0}^{r}, H^{r}\right)$ on the negative semi-axis can be computed via the eigenvalue counting function $N^{r}(\lambda)$ for the Schrödinger operator $H^{r}$ (cf. [10]),

$$
\xi^{r}(\lambda)=-N^{r}(\lambda), \quad \lambda<0 .
$$

For $\lambda \geq 0$, the function $\xi$ admits the representation in terms of the phase shift $\delta$, associated with the potential $V$ (see, e.g., [2], [4],[6], [15]),

$$
\xi(\lambda)=-\pi^{-1} \delta(\sqrt{\lambda}), \quad \lambda \geq 0 .
$$

On the other hand, on the positive semi-axis the function $\xi^{r}$ can be represented as

$$
\xi^{r}(\lambda)=\left[\pi^{-1} r \sqrt{\lambda}\right]-\left[\pi^{-1} r \sqrt{\lambda}+\pi^{-1} \delta^{r}(\sqrt{\lambda})\right], \quad \lambda \geq 0, \quad r>0,
$$

where $\delta^{r}$ is the phase shift associated with the cut off potential $V^{r}$ given by

$$
V^{r}(x)= \begin{cases}V(x), & 0 \leq x \leq r, \\ 0, & x>r .\end{cases}
$$

To prove (3.4) one observes that

$$
\xi^{r}(\lambda)=N_{0}^{r}(\lambda)-N^{r}(\lambda), \quad \lambda \geq 0, \quad r>0,
$$

where $N_{0}^{r}$ is the eigenvalue counting function for the Schrödinger operator $H_{0}^{r}$ on the finite interval.

Since

$$
N_{0}^{r}(\lambda)=\left[\pi^{-1} r \sqrt{\lambda}\right], \quad \lambda \geq 0,
$$

and $N^{r}$ can be represented as

$$
N^{r}(\lambda)=\left[\pi^{-1} r \sqrt{\lambda}+\pi^{-1} \delta^{r}(\sqrt{\lambda})\right], \quad \lambda \geq 0,
$$

(by the well known counting principle that is a direct consequence of the Sturm oscillation theorem (see, e.g., [13, Ch. II, Sec. 6])), one gets (3.4).

In particular, since the counting functions $N, N^{r}$, and $N_{0}^{r}$ are continuous from the left and under hypothesis (1.3) the phase shift $\delta$ is continuous on $[0, \infty)$, equations (3.1), (3.3) and (3.4), (3.6) determine the left-continuous representatives for the spectral shift functions $\xi$ and $\xi^{r}$, respectively.

To prove the first assertion (1.4) of the theorem we proceed as follows.

Given a continuous function $g$ with compact support, one splits the left hand side of (1.4) into two parts

$$
\int_{-\infty}^{\infty} \xi^{r}(\lambda) g(\lambda) d \lambda=I_{r}+I I_{r}, \quad r>0
$$

where

$$
I_{r}=\int_{-\infty}^{0} \xi^{r}(\lambda) g(\lambda) d \lambda \quad \text { and } \quad I I_{r}=\int_{0}^{\infty} \xi^{r}(\lambda) g(\lambda) d \lambda
$$


First we prove that

$$
\lim _{r \rightarrow \infty} I_{r}=\int_{-\infty}^{0} \xi(\lambda) g(\lambda) d \lambda .
$$

Assume that the half-line Schrödinger operator $H$ has $m, m \geq 0$, negative eigenvalues denoted by

$$
\lambda_{1}(H)<\lambda_{2}(H)<\ldots<\lambda_{m}(H) .
$$

Then in accordance with a result in [1], there exists a $r_{0}$ such that for all $r>r_{0}$ the operator $H^{r}$ has $m^{r}, m^{r} \geq m$, negative eigenvalues

$$
\lambda_{1}\left(H^{r}\right)<\lambda_{2}\left(H^{r}\right)<\ldots<\lambda_{m^{r}}\left(H^{r}\right), \quad r>0,
$$

and, in addition,

$$
\lim _{r \rightarrow \infty} \lambda_{k}\left(H^{r}\right)=\lambda_{k}(H), \quad k=1,2, \ldots, m .
$$

Moreover, for any $\varepsilon>0$ there exists $r(\varepsilon)$ such that

$$
-\varepsilon<\lambda_{k}\left(H^{r}\right)<0, \quad k=m+1, \ldots, m^{r}, \quad r>r(\varepsilon) .
$$

Taking into account (3.2), from (3.9) one gets that

$$
\lim _{r \rightarrow \infty} \xi^{r}(\lambda)=\xi(\lambda), \quad \lambda \in(-\infty, 0) \backslash \operatorname{spec}_{d}(H),
$$

where $\operatorname{spec}_{d}(H)$ denotes the discrete (negative) spectrum of the operator $H$.

To deduce (3.8) from (3.10) it suffices to apply a Bargmann-type estimate [14] that provides an upper bound for the number $m^{r}$ of the negative eigenvalues of the operator $H^{r}$ as follows

$$
m^{r} \leq \int_{0}^{r} x V_{-}(x) d x \leq \int_{0}^{\infty} x V_{-}(x) d x<\infty, \quad r>0,
$$

where $V_{-}$stands for the negative part of the potential

$$
V_{-}(x)= \begin{cases}-V(x), & V(x)<0 \\ 0, & \text { otherwise }\end{cases}
$$

Indeed, since

$$
\left|\xi^{r}(\lambda)\right|=N^{r}(\lambda) \leq m^{r} \leq \int_{0}^{\infty} x V_{-}(x) d x \leq \int_{0}^{\infty}(1+x)|V(x)| d x<\infty, \quad \lambda<0,
$$

equality (3.8) follows from (3.10) by the dominated convergence theorem.

Next we prove that

$$
\lim _{r \rightarrow \infty} I I_{r}=\int_{0}^{\infty} \xi(\lambda) g(\lambda) d \lambda
$$

To get (3.12) it suffices to show that for every sequence $\left\{r_{n}\right\}_{n=1}^{\infty}$ of non-negative numbers $r_{n}$ such that $\lim _{n \rightarrow \infty} r_{n}=\infty$ the equality

$$
\lim _{n \rightarrow \infty} \int_{0}^{\infty} \xi^{r_{n}}(\lambda) g(\lambda) d \lambda=\int_{0}^{\infty} \xi(\lambda) g(\lambda) d \lambda
$$

holds.

From (3.4) one derives that

$$
\int_{0}^{\infty} \xi^{r}(\lambda) g(\lambda) d \lambda=\int_{0}^{\infty}\left(\left[\pi^{-1} r \sqrt{\lambda}\right]+\left[\pi^{-1} r \sqrt{\lambda}-\pi^{-1} \delta^{r}(\sqrt{\lambda})\right]\right) g(\lambda) d \lambda, \quad r>0,
$$


and therefore, after a change of variables, one obtains that

$$
\int_{0}^{\infty} \xi^{r_{n}}(\lambda) g(\lambda) d \lambda=\int_{0}^{1}\left(\left[t_{n} \lambda\right]-\left[t_{n} \lambda+f_{n}(\lambda)\right]\right) \widehat{g}(\lambda) d \lambda, \quad n \in \mathbb{N},
$$

where $t_{n}=\pi^{-1} r_{n}, \widehat{g}(\lambda)=2 \lambda g\left(\lambda^{2}\right), \lambda \geq 0$, and the sequence of functions $\left\{f_{n}\right\}_{n=1}^{\infty}$ is given by

$$
f_{n}(\lambda)=\pi^{-1} \delta^{r_{n} \pi}(\lambda), \quad n \in \mathbb{N} .
$$

Without loss of generality (by rescaling) one may assume that $g(\lambda)=0$ for $\lambda>1$ and hence to prove (3.13) it remains to check that the sequence of functions (3.14) satisfies the hypotheses of Lemma2.2 with

$$
f(\lambda)=\pi^{-1} \delta(\lambda), \quad \lambda>0 .
$$

Indeed, from the phase equation ([7], p. 11, Eq. (13); [5])

$$
\frac{d}{d r} \delta^{r}(k)=-k^{-1} V(r) \sin ^{2}\left(k r+\delta^{r}(k)\right), \quad k>0, \quad r>0,
$$

one easily concludes that

$$
\left|\delta^{r}(\sqrt{\lambda})-\delta(\sqrt{\lambda})\right| \leq \frac{1}{\sqrt{\lambda}} \int_{r}^{\infty}\left|V\left(r^{\prime}\right)\right| d r^{\prime}, \quad \lambda>0, \quad r>0,
$$

and therefore

$$
\left|f_{n}(\lambda)-f(\lambda)\right| \leq \frac{1}{\pi \lambda} \int_{\pi r_{n}}^{\infty}\left|V\left(r^{\prime}\right)\right| d r^{\prime}, \quad \lambda>0, \quad n \in \mathbb{N} .
$$

which proves that

$$
\lim _{n \rightarrow \infty} f_{n}(\lambda)=f(\lambda), \quad \lambda>0,
$$

and that the convergence in (3.18) takes place uniformly on every compact subset of the semi-open interval $(0,1]$.

Moreover, the following bound

$$
\left|f_{n}(\lambda)\right| \leq \sup _{\lambda>0}|f(\lambda)|+\frac{1}{\pi \lambda} \int_{0}^{\infty}\left|V\left(r^{\prime}\right)\right| d r^{\prime}, \quad \lambda>0, \quad n \in \mathbb{N},
$$

holds and therefore the sequence (3.14) has a $\widehat{g}$-integrable majorante.

Finally, under the short range hypothesis (1.3) the phase shift $\delta(\lambda)$ is a continuous function on $(0, \infty)$ and by the Levinson Theorem,

$$
\lim _{\lambda \downarrow 0} \delta(\sqrt{\lambda})=\pi m, \quad m \in \mathbb{Z}_{+},
$$

if there is no zero-energy resonance and

$$
\lim _{\lambda \downarrow 0} \delta(\sqrt{\lambda})=\pi\left(m+\frac{1}{2}\right), \quad m \in \mathbb{Z}_{+}, \quad \text { otherwise. }
$$

Therefore, the function $f$ is continuous on $[0,1]$ and hence it is Riemann integrable.

Now one can apply Lemma2.2 to conclude that

$$
\begin{aligned}
\lim _{n \rightarrow \infty} \int_{0}^{\infty} \xi^{r_{n} \pi}(\lambda) g(\lambda) d \lambda & =-\int_{0}^{1} f(\lambda) \widehat{g}(\lambda) d \lambda=-\frac{1}{\pi} \int_{0}^{1} \delta(\lambda) 2 \lambda g\left(\lambda^{2}\right) d \lambda \\
& =-\frac{1}{\pi} \int_{0}^{1} \delta(\sqrt{\lambda}) g(\lambda) d \lambda=\int_{0}^{\infty} \xi(\lambda) g(\lambda) d \lambda,
\end{aligned}
$$

which proves 3.13 . 
The proof of (1.4) is complete.

Now, we will prove the second statement (1.5) of the theorem.

First we remark that equation (3.10) immediately implies (1.5) for $\lambda<0, \lambda \notin \operatorname{spec}_{d}(H)$. In order to prove convergence (1.5) for a fixed $\lambda>0$, we again use equation (3.4) to obtain

$\frac{1}{r} \int_{0}^{r} \xi^{r}(\lambda) d r=\frac{1}{r} \int_{0}^{r}\left[\pi^{-1} r \sqrt{\lambda}\right]-\left[\pi^{-1} r \sqrt{\lambda}+\pi^{-1} \delta^{r}(\sqrt{\lambda})\right] d r, \quad \lambda>0, \quad r>0$.

After the change of variables $x=\pi^{-1} r \sqrt{\lambda}$, we get

$$
\frac{1}{r} \int_{0}^{r} \xi^{r}(\lambda) d r=\frac{\pi}{\sqrt{\lambda} r} \int_{0}^{\frac{r \sqrt{\lambda}}{\pi}}\left([x]-\left[x+\pi^{-1} \delta^{\pi x / \sqrt{\lambda}}(\sqrt{\lambda})\right]\right) d x, \lambda>0, r>0 .
$$

From (3.16) it follows that the function $h$ given by

$$
h(x)=\delta^{\pi x / \sqrt{\lambda}}(\sqrt{\lambda}), \quad x>0, \quad \lambda>0,
$$

is a bounded function and that

$$
\lim _{x \rightarrow \infty} h(x)=\delta(\sqrt{\lambda}), \quad \lambda>0 .
$$

Hence, applying Corollary 2.3 one concludes that

$$
\lim _{r \rightarrow \infty} \frac{\pi}{\sqrt{\lambda} r} \int_{0}^{\frac{r \sqrt{\lambda}}{\pi}}\left([x]-\left[x+\pi^{-1} \delta^{\pi x / \sqrt{\lambda}}(\sqrt{\lambda})\right]\right) d x=-\delta(\sqrt{\lambda}), \lambda>0,
$$

and, therefore,

$$
\lim _{r \rightarrow \infty} \frac{1}{r} \int_{0}^{r} \xi^{r}(\lambda) d r=-\pi^{-1} \delta(\sqrt{\lambda}), \quad \lambda>0,
$$

which together with (3.3) proves (1.5) for $\lambda>0$.

Finally, to prove (1.5) for $\lambda=0$ in the case of the absence of a zero energy resonance, notice that the corresponding Jost function $\mathcal{F}$ does not vanish on the closed semi-interval $[0, \infty)$. Since

$$
\lim _{r \rightarrow \infty} \mathcal{F}^{r}(k)=\mathcal{F}(k), \quad k \geq 0,
$$

it is obvious that $\mathcal{F}^{r}(k)$ is not zero for $k \in[0, \infty)$ for all $r$ large enough and hence

$$
\lim _{r \rightarrow \infty} \delta(r, 0)=\delta(0)
$$

for $\delta(r, k)=\arg \left(\mathcal{F}^{r}(k)\right)$ and $\delta(k)=\arg (\mathcal{F}(k))$.

Now equations (3.21), 3.23), and Corollary 2.3 imply

$$
\lim _{r \rightarrow \infty} \frac{1}{r} \int_{0}^{r} \xi^{r}(0) d r=-\pi^{-1} \delta(0) .
$$

By the Levinson Theorem (see (3.20) $)$, the quantity $\pi^{-1} \delta(0)$ coincides with the number $N(0)$ of (negative) eigenvalues of the Schrödinger operator $H$. Since

$$
\xi(0)=\lim _{\varepsilon \downarrow 0} \xi(-\varepsilon)=-N(0),
$$

one concludes that

$$
\lim _{r \rightarrow \infty} \frac{1}{r} \int_{0}^{r} \xi^{r}(0) d r=\xi(0)
$$

which completes the proof of Theorem 1

Acknowledgments We would like to thank Fritz Gesztesy and Vadim Kostrykin for useful discussions on this topic. 


\section{REFERENCES}

[1] P. B. Bailey, W. N. Everitt, J. Weidmann and A. Zettle, Regular approximations of singular Sturm-Liouville problems, Results in Mathematics, 23 (1993), 3-22.

[2] M. Sh. Birman and M. G. Krein, On the theory of wave operators and scattering operators, Dokl. Akad. Nauk SSSR, 144 (1962), 475-478; English transl. in Soviet Math. Dokl. 3 (1962).

[3] M. Sh. Birman and A. B. Pushnitski, Spectral shift function, amazing and multifaceted. Dedicated to the memory of Mark Grigorievich Krein (1907-1989), Integral Equations and Operator Theory, 30 (1998), no. 2, 191-199.

[4] M. Sh. Birman and D. R. Yafaev, The Spectral Shift Function. The Work of M. G. Krein and its Further Development, St. Petersburg Math J., 4 (1993), no. 5, 833-870.

[5] E. Brüning and F. Gesztesy, Continuity of wave and scattering operators with respect to interactions, J. Math. Phys., 24 (1983), no. 6, 1516-1528.

[6] V. S. Buslaev and L. D. Faddeev, On trace formulas for a singular differential Sturm-Liouville operator, Dokl. Akad. Nauk SSSR, 132 (1960), 13-16; English transl. in Soviet Math. Dokl. 1 (1960).

[7] F. Calogero, Variable Phase Approach to Potential Scattering, Academic Press, New York, London, (1967).

[8] K. Chadan and P. C. Sabatier, Inverse Problems in Quantum Scattering Theory, Springer-Verlag, New York, (1977).

[9] R. Geisler, V. Kostrykin, and R. Schrader, Concavity Properties of Krein's Spectral Shift Function, Reviews in Mathematical Physics, 7 (1995), 161-181.

[10] M. G. Krein, On a trace formula in perturbation theory, Matem. Sbornik, 33 (1953), 597-626 (in Russian).

[11] M. G. Krein, On perturbation determinants and the trace formula for unitary and selfadjoint operators, Dokl. Akad. Nauk SSSR, 144 (1962), 268-271; English transl. in Soviet Math. Dokl. 3 (1962).

[12] R. G. Newton, Scattering Theory of Waves and Particles, Springer - Verlag, New York, (1982).

[13] L. Pastur and A. Figotin, Spectra of Random and Almost-Periodic Operators, Springer-Verlag, Berlin Heidelberg, (1992).

[14] K. M. Schmidt, A short proof for Bargmann-type inequalities, Proc. R. Soc. Lond. A, 458 (2002), 28292832.

[15] D. R. Yafaev, Mathematical Scattering Theory. General Theory, American Mathematical Society, (1991).

Department of Mathematics, University of ARIZONa, PO BoX 210089, TuCson, AZ 857210089 , USA

E-mail address: borovykv@math.arizona.edu

Department of Mathematics, University of Missouri, Columbia, Missouri 63211, USA

E-mail address: makarovk@missouri.edu 